\title{
Zur Anerkennung als sog. „Aufbaupraxis“ und dem Anspruch einer unterdurchschnittlich abrechnenden Praxis im Rahmen des Honorarverteilungsrechts
}

\section{Einführung}

Wenn es um die Vergütung in der vertragsärztlichen Versorgung geht, dann stoßen 2 Interessenlagen aufeinander, die nicht mehr zu vereinbaren sind. Während der niedergelassene, vertragsärztlich tätige Radiologe hohe Investitionen für seine Praxis zu tätigen hat und ein berechtigtes Interesse an einer sicheren Prognose der zukünftigen Einnahme wünscht und aus Sicht einer finanzierenden Bank benötigt, verändern der Gesetzgeber, der Bewertungsausschuss und die Kassenärztlichen Vereinigungen in immer kürzen Abständen die Honorarverteilung bis hin zur Honorarsystematik, um die Vergütungsvorgaben im Gesundheitswesen nicht besser, sondern allenfalls restriktiver $\mathrm{zu}$ gestalten. Dies hat zur Folge, dass in bestimmten Bereichen die wirtschaftliche Planbarkeit für radiologische Praxen, insbesondere für den wirtschaftlichen Einsatz von Großgeräten nicht mehr gegeben ist. In seiner Entscheidung vom 17.07.2013 hat das Bundessozialgericht (Az.: B 6 KA 44/12 $\mathrm{R})$ über mehrere Rechtsfragen entschieden, die die Wirtschaftlichkeit einer radiologischen, vertragsärztlichen Praxis mehr als infrage stellen. Ein Fortbestand der honorarverteilungsrechtlichen Regelungen und dieser Rechtsprechung gefährdet die Sicherstellung der vertragsärztlichen Versorgung durch Radiologen.

\section{Sachverhalt}

$\nabla$

Die Klägerin in dem Rechtsstreit, der dem BSG zur Entscheidung vorlag, war eine Berufsausübungsgemeinschaft (BAG) aus Fachärzten für Radiologie. Die Praxis war mit einem Computertomografen (CT) und einem Magnetresonanztomografen (MRT) ausgestattet. Die BAG war seit 2004 vertragsärztlich tätig und bestand aus 2 Radiologen. Anfang 2008 trat eine weitere Radiologin als Partnerin in die BAG ein. Sie war bereits seit dem Jahre 2005 zur vertragsärztlichen Versorgung zugelassen und zuvor in einer Einzelpraxis tätig gewesen.
Die beklagte Kassenärztliche Vereinigung wies der BAG für das Quartal III/2009 ein Regelleistungsvolumen (RLV) von 3700,09€ zu. Dem lag die Multiplikation der RLV-relevanten Fallzahl aus dem Quartal III/2008-90 Behandlungsfälle mit dem arztgruppenspezifischen Fallwert zugrunde, zuzüglich eines $10 \%$ igen BAG-Aufschlags. Später korrigierte die Kassenärztliche Vereinigung die Zuweisung auf ein RLV in Höhe von 5860,69€. Das ausgezahlte Honorar für das Quartal III/2009 belief sich auf 9244,12€; der Berechnung lag die vorjährige Zahl von 90 Fällen zugrunde; die tatsächliche Fallzahl der BAG im Quartal III/2009 belief sich auf 371 Behandlungsfälle. Die BAG war mit dieser Vergütung unzufrieden und klagte bis zum BSG. Nach einem zunächst teilweisen Erfolg der Klage vor dem Hessischen Landessozialgericht blieb die Klage am Ende vor dem BSG erfolglos.

\section{Regelungen zur Honorarvertei- lung nach § 87b SGB V \\ $\nabla$}

Nach Auffassung des BSG hatte der Bewertungsausschuss nach $\S 87 \mathrm{~b}$ Abs. 4 Sätze 1 und 2 SGB V das Verfahren zur Berechnung und zur Anpassung der RLV zu bestimmen. Nach dem Scheitern einer Einigung im Bewertungsausschuss schuf der erweiterte Bewertungsausschuss ( $§ 87$ Abs. 4 SGB V) durch Beschluss vom 27./28.08.2008 im Teil F Nr 3.2.1, 3.4 und 3.5 sog. Basisregelungen: Diese sahen vor, dass für die Bemessung des RLV die Fallzahl im Vorjahresquartal maßgebend war (Nr. 3.2.1 Satz 2) und dass Ausnahmen hiervon bei Vorliegen bestimmter Voraussetzungen geregelt werden können $(\mathrm{Nr}$ 3.4). Ferner war geregelt, dass die Kassenärztlichen Vereinigungen zusammen mit den Krankenkassen im Gesamtvertrag ergänzende Regelungen für Neuzulassungen und Kooperationsumwandlungen „Zur Sicherung einer angemessenen Vergütung“ vertraglich beschließen können. Die Partner der Gesamtverträge im Bezirk der beklagten Kassenärztlichen Vereinigung Hessen konzentrierten sich bei der

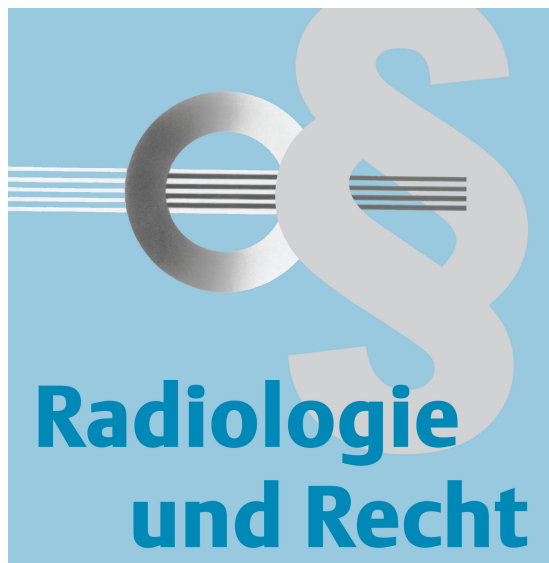

Ausformung des Honorarverteilungsvertrages darauf, die hier relevanten zwingenden bundesgesetzlichen Regelungen zu wiederholen. Sie trafen nur wenige ergänzende Regelungen, die speziell auf Aufbau- und/oder sonstige unterdurchschnittlich abrechnende Praxen ausgerichtet waren.

\section{Wachstumsmöglichkeiten unter- durchschnittlich abrechnender Praxen \\ $\nabla$}

In seinem Urteil musste sich das BSG erneut mit den Wachstumsmöglichkeiten einer unterdurchschnittlich abrechnenden Praxis auseinandersetzen. Dabei führt das BSG in ständiger Rechtsprechung aus, dass umsatzmäßig unterdurchschnittlich abrechnende Praxen die Möglichkeit haben müssen, zumindest den durchschnittlichen Umsatz der Arztgruppe zu erreichen (stRspr, zusammenfassend BSG SozR 4-2500 § $85 \mathrm{Nr} 45 \mathrm{RdNr}$. 23-33 und Nr. 50 RdNr. 14-16, jeweils mwN; BSG vom 05.06.2013, Az.: B 6 KA $32 / 12$ R). Dem Vertragsarzt müsse - wegen seines Rechts auf berufliche Entfaltung unter Berücksichtigung der sog Honorarverteilungsgerechtigkeit - die Chance bleiben, durch Qualität und Attraktivität seiner Behandlung oder auch durch eine bessere Organisation seiner Praxis neue Patienten für sich zu gewinnen und so legitimerweise seine Position im Wettbewerb mit den Berufskollegen zu verbessern. Daher sei allen Praxen mit unterdurchschnittlichen Umsätzen die Möglichkeit einzuräumen, durch Umsatzsteigerung jedenfalls bis zum Durchschnittsumsatz der Fachgruppe aufzuschließen und damit ihre Praxis zu einer mit typischen Umsätzen auszubauen (stRspr, z. B. BSG SozR 4-2500 § 85 Nr. 50 Rd-Nr. 14; BSG vom 05.06.2013, Az. B 6 KA 32/12 R). 
In zeitlicher Hinsicht hat das BSG diese Vorgabe dahingehend konkretisiert, dass Praxen in der Aufbauphase - die nach der Rechtsprechung von den Kassenärztlichen Vereinigungen auf einen Zeitraum von 3, 4 oder 5 Jahren bemessen werden kann - die Steigerung ihres Honorars auf den Durchschnittsumsatz sofort möglich sein muss, während dies anderen, noch nach der Aufbauphase unterdurchschnittlich abrechnenden Praxen jedenfalls innerhalb von 5 Jahren ermöglicht werden muss (BSG SozR 4-2500 § $85 \mathrm{Nr} 50 \mathrm{RdNr}$ $15 \mathrm{mwN}$ ). Die Bemessung des Zeitraums der Aufbauphase erfolgt im Honorarverteilungsvertrag durch dessen Vertragspartner bzw. in der Satzung über die Honorarverteilung (heute: Honorarverteilungsmaßstab) durch die Kassenärztliche Vereinigung.

Die dargestellten Grundsätze hatten auch im Zeitraum 2009-2011, in dem die Honorarverteilung durch die Regelungen des $\S 87 b$ SGB V iVm den Vorgaben des Bewertungsausschusses geprägt war, Geltung. Dies folge, so das BSG, insbesondere daraus, dass sich der sog. Wachstumsanspruch für unterdurchschnittlich abrechnende Praxen - vor allem - auf den Grundsatz der Honorarverteilungsgerechtigkeit und damit auf Artikel 3 Abs. 1 (Grundrecht auf allgemeine Gleichbehandlung) und 12 Abs. 1 GG (Grundrecht auf Schutz der Berufsausübungsfreiheit) stütze. Dadurch komme ihm sowohl gegenüber Landesals auch gegenüber Bundesrecht Geltungskraft zu, sodass er auch gegenüber der Rechtssetzung des Bewertungsausschusses Wirkung entfalte und bei der Auslegung der von diesem geschaffenen Rechtsnormen zu beachten sei. Dieses Ergebnis stimme auch mit der Aussage im Urteil des BSG vom 03.02.2010 (SozR 4-2500 § $85 \mathrm{Nr} 50$ ) überein, dass Beschlüsse des Bewertungsausschusses für sich genommen keine Benachteiligung von unterdurchschnittlich abrechnenden Praxen bzw. von Aufbaupraxen rechtfertigen könnten.

\section{Voraussetzungen einer „Aufbau- praxis"6 \\ $\nabla$}

In dem Ausgangsfall stand die Frage zur Entscheidung an, ob durch die Aufnahme der Partnerin in die BAG eine Aufbaupraxis entstanden war oder nicht. Im Ergebnis lehnt das BSG dies zulasten der klagenden BAG ab.
Die genaue Bestimmung des Zeitraums des Aufbaus einer Praxis, bei der es sich um eine Erstzulassung - sog. Anfängerpraxis - oder um eine Neuzulassung nach vorheriger vertragsärztlicher Tätigkeit in einem anderen Planungsbereich handeln könne, sei der Regelung im Honorarverteilungsmaßstab der Kassenärztlichen Vereinigung vorbehalten. Es könne festgelegt werden, ob der Anspruch auf sofortige Honorarsteigerung bis zum Durchschnittsumsatz der Arztgruppe für einen Zeitraum von 3, 4 oder 5 Jahren bestehen solle.

Der Eintritt eines weiteren Arztes in eine BAG stellt nach Ansicht des BSG keine Neuaufnahme vertragsärztlicher Tätigkeit dar. Bei der Neuformierung einer BAG durch Austritt oder Neueintritt eines Partners geht das BSG, im Einklang mit der Rechtsprechung des Bundesgerichtshofes davon aus, dass die Gesellschaft bürgerlichen Rechts, die Partnerschaftsgesellschaft und gleichermaßen auch die BAG unverändert fortbestehen (BSG SozR 4-1500 § 141 Nr. 1 RdNr. 17; Urteile vom 17.10.2012, Az.: B 6 KA 39/11 R, B 6 KA 41/11 R, B 6 KA 42/11 R und B 6 KA 44/11 $\mathrm{R}$; entsprechend $\mathrm{BGH}$, Beschluss vom 02.12.2010, Az.: V ZB 84/10). Dies gelte beim Eintritt eines neuen Partners unabhängig davon, wie lange dieser schon praktiziert habe. Eine BAG könne sich nicht durch Aufnahme eines jungen Partners „verjüngen“ und so die Eigenschaft als Aufbaupraxis länger als 5 Jahre - oder gar durch regelmäßige Neueintritte junger Partner fortwährend - behalten. Vielmehr müssten sich auf der Grundlage der Rechtsprechung des BSG die BAG und der Neueintretende darüber im Klaren sein, dass dieser sich durch den Eintritt in die BAG in diese einbindet. Damit könne der Verlust von bestimmten Vorteilen verbunden sein, wie etwa der bisherigen Position seiner Einzelpraxis als Aufbaupraxis, wenn nämlich die BAG, in die er eintrete, keine Aufbaupraxis mehr sei.

Das BSG hat erkannt, dass infolge der vorstehenden Ausführungen ein Verstoß gegen die grundrechtlich geschützte allgemeine Gleichbehandlung vorliegen kann und warf die Frage auf, ob bzw. in welcher Weise bei Eintritt eines Arztes in eine BAG die ihr zuzurechnende, für das RLV maßgebliche Fallzahl zu berechnen sei.

Das BSG stellte dann allerdings fest, dass diese Frage keiner Erörterung bedürfe. Die Kassenärztliche Vereinigung dürfe im 1.
Jahr nach dem Eintritt eines Arztes in eine BAG das RLV für die BAG nicht allein nach deren Fallzahl im jeweiligen Vorjahresquartal berechnen, sondern müsse eine zusätzliche Fallzahl für das neu eintretende Mitglied berücksichtigen - was z.B. entweder durch eine Erhöhung der Fallzahl der BAG entsprechend dem Personenzuwachs in der BAG oder durch Hinzurechnung der vom Eintretenden zuvor erbrachten Fallzahlen erfolgen könne und was eventuell normativ-schematisch durch die Kassenärztliche Vereinigung vorgegeben oder an der Gestaltung des konkreten Falles ausgerichtet werden könne. Eine Festlegung vermied das BSG jedoch und überlässt es der Kassenärztlichen Vereinigung, eine grundgesetzkonforme Regelung in den Honorarverteilungsmaßstab aufzunehmen.

\section{Sog. „Einjähriges Moratorium“ $\nabla$}

Die klagende BAG hatte daneben gerügt, dass die Honorarsystematik verlange, dass die Ärzte 1 Jahr arbeiten müssten, für die Leistungen indes keine Vergütung erhalten würden.

Das BSG folge dieser Auffassung nicht, sondern verwies darauf, dass das sog. 1-jährige Moratorium seine Grundlage in der Sonderregelung des $\S$ 87b Abs. 4 Satz $1 \mathrm{iVm}$ Abs. 2 Satz 2 SGB V finde, wonach der Bewertungsausschuss zu Vorgaben für den Inhalt der RLV und zur Bestimmung des Verfahrens für deren Berechnung ermächtigt sei. Der Bewertungsausschuss habe dabei ein gewisses Maß an Gestaltungsfreiheit, wie das BSG mehrfach im Zusammenhang mit anderen, dem Bewertungsausschuss eingeräumten, Rechtssetzungsbefugnissen festgestellt hat (stRspr, vgl zB BSGE 105, 236 = SozR 4-2500 § 85 Nr.. 53, RdNr. 21 ff, 26, 29; BSGE 106, 56 = SozR 4-2500 § $85 \mathrm{Nr}$. 54, RdNr. 20 f; vgl auch BSG vom 9.5.2012, Az.: B 6 KA 30/11 R; vgl ferner Urteil vom 06.02.2013, Az.: B 6 KA 13/12 R). Insoweit sei der Bewertungsausschuss frei, bei der Ausgestaltung der Regelungen für die RLV auch andere legitime Ziele zu verfolgen, wie z.B. Anreize für Fallzahlvermehrungen zur Honorarsteigerung zu mindern und dadurch die Gesamthonorarsituation zu stabilisieren sowie die Kalkulierbarkeit der Einnahmen aus vertragsärztlicher Tätigkeit zu verbessern. Das BSG hat dazu ausgeführt, dass es für diesen Wachstumsanspruch nicht ausreiche, den Fachgruppendurchschnitt irgendwie und ir- 
gendwann erreichen zu können, sondern dass es dem Vertragsarzt vielmehr möglich sein muss, die Steigerung bis zum Durchschnitt „in effektiver Weise“ und in „realistischer Weise“ zu erreichen (BSG SozR 4-2500 § $85 \mathrm{Nr} 45$ ). Dies erfordere allerdings nicht die Möglichkeit einer kontinuierlicher Steigerung, sondern es komme lediglich auf das Ergebnis - die Möglichkeit, den Durchschnittsumsatz zu erreichen - an (BSG SozR 4-2500 § $85 \mathrm{Nr}$ 45; SozR 4-2500 § $85 \mathrm{Nr} 50$ ). Praxen mit unterdurchschnittlichem Umsatz müssten nicht von jeder Begrenzung des Honorarwachstums verschont werden (BSGE 92, 10 = SozR 4-2500 § 85 Nr 5). Ein Anspruch darauf, dass die Gesamtzahl der in einem Quartal behandelten Fälle jeweils sogleich dem RLV für dieses Quartal zugrunde gelegt würde, bestehe nicht.

Verfassungsrechtlich hätte das BSG der Frage nachgehen können und müssen, ob vor dem Hintergrund der grundrechtlich geschützten Berufsausübung nicht die Kassenärztliche Vereinigung die Begrenzung des Wachstums rechtfertigen können muss. Der Anspruch des Vertragsarztes auf eine gleiche Vergütung besteht bereits aufgrund des Grundrechts auf Gleichbehandlung nach Art. 3 Abs. 1 GG. Immer wieder geht es in diesem Kontext um die Frage, ob der Radiologe einen Anspruch auf eine bestimmte Genehmigung haben muss oder ob nicht vielmehr die Kassenärztliche Vereinigung oder in anderen Zusammenhängen der Zulassungsausschuss einer Rechtsgrundlage bedürfen, die Rechte des Radiologen einzuschränken.

Im Weiteren führte das BSG aus, dass Bestimmungen nicht ausgeschlossen sind, die ein Honorarwachstum innerhalb eines gewissen Zeitraums unterbinden, (BSG SozR 4-2500 § 85 Nr 45), sofern die Praxen in der nach Ablauf des Moratoriums verbleibenden Zeit noch die „effektive, dh realistische, Möglichkeit“ haben, den Durchschnittsumsatz zu erreichen.

Das BSG geht in seiner Entscheidung nicht auf die Frage ein, ob nicht bereits aufgrund des Überweisungserfordernisses nach $\S 13$ Abs. 4 BundesmantelvertragÄrzte (BMV-Ä) zumindest eine erste Beschränkung des - ungehemmten - Praxiswachstums vorliegt und in der Folge eine übermäßige Tätigkeitsausweitung unmöglich ist, sodass der Sinn eines Moratoriums jedenfalls nicht mehr in der übermäßigen Tätigkeitsausweitung liegen könnte.
Das LSG Berlin-Brandenburg hat diese Auffassung erst jüngst in einem Urteil vom 20.02.2013 (Az.: L 7 KA 60/11) bestätigt und folgendes festgestellt:

„Demgegenüber unterliegen Arztgruppen der sog. Methodenfächer, wie z. B. Laborärzte, Nuklearmediziner, Pathologen und Radiologen, dem Überweisungsvorbehalt (§ 13 IV Bundesmantelvertrag-Ärzte (BMV-Ä) bzw. § 7 IV Arzt-Ersatzkassenvertrag (EKV)) und den Beschränkungen des Zielauftrags ( $§ 24$ VII 2 Nr. 2 BMV-Ä und $\S 27$ VII 1 Nr. 2 EKV). Beides verhindert weitgehend, dass Mitglieder dieser Arztgruppen allein durch eigenes Zutun ihre Leistungsmenge ausweiten können (s.a. Wigge NZS 05, 176).“

Davon ausgehend, dass eine Praxis grundsätzlich eine Zeit lang an ihrem Praxisund Honorierungsumfang festgehalten werden dürfe, so das BSG, sei das Moratorium von 1 Jahr, soweit es nach Abschluss der Aufbauphase greife, nicht zu beanstanden. Durchgreifende Bedenken ergäben sich auch nicht bei Berücksichtigung des Wachstumsanspruchs unterdurchschnittlich abrechnender Praxen. Dem Arzt bliebe es unbenommen, mit seiner Praxis durch eine Fallzahlsteigerung ein höheres RLV für das Folgejahr zu erzielen und so - wie es den unterdurchschnittlich abrechnenden Praxen möglich sein muss - im Gesamtzeitraum von 5 Jahren den Durchschnittsumsatz der Fachgruppe zu erreichen. Das BSG widersprach sodann dem Hessischen Landessozialgericht, dass die Regelungen als unvertretbar und unverhältnismäßig beurteilt hatte und verwies darauf, dass Honorarsonderregelungen wie Härteklauseln zu berücksichtigen seien.

\section{Härtefallregelung \\ $\nabla$}

Zunächst ist nach Auffassung des BSG zu beachten, dass im Allgemeinen auch bei Honorarregelungen Regelungen für den Umgang mit Härtefällen bestehen und dass bei Fehlen einer ausdrücklichen Härteklausel diese in die Honorarbestimmungen hineinzuinterpretieren sei (BSG SozR 4-2500 § 85 Nr 66 RdNr 28-30; BSG MedR 2012, 413). Bei Mitberücksichtigung der Härteklausel könne die Bestimmung, die einen Mehrverdienst durch Fallzahlerhöhungen für ein ganzes Jahr weitgehend ausschlösse, zumal deshalb nicht als „unvertretbar und unverhältnismäßig“ bewertet werden, weil die Härteklausel ge- rade die Funktion habe, unverhältnismäßigen Nachteilen vorzubeugen, was insbesondere für atypische Konstellationen von Bedeutung sei.

Nach dem bisherigen Verlauf der Entscheidung überrascht es nicht weiter, dass das BSG zwar die Härteklausel anführt, diese auf den zu entscheidenden Fall letztlich jedoch nicht anwendet.

Ein von der BAG nicht zu vertretender, als Härte in Betracht kommender Umstand ergebe sich, so das BSG, nicht bei Einbeziehung des Umstandes, dass die spätere geringe Honorierung im Quartal III/2009 ihren Ursprung schon vorher, nämlich in der geringen Fallzahl im Quartal III/2008 verbunden mit dem 1-jährigen Moratorium für die Bewirkung einer Erhöhung des RLV durch Fallzahlerhöhungen -, hatte. Zwar habe die BAG während ihrer vertragsärztlichen Tätigkeit im Quartal III/2008 noch nicht wissen können, dass sich die dabei erzielte Fallzahl begrenzend auf ihr Honorar für das Quartal III/2009 auswirken würde (der Beschluss des Erweiterten Bewertungsausschusses vom 27./28.8.2008 wurde erst gegen Ende des Quartals bekannt gemacht). Dieses Nichtwissen sei aber nicht schutzwürdig. Vielmehr sei es dem unternehmerischen Risiko des Vertragsarztes zuzurechnen, wie er seine Praxistätigkeit gestalte, insbesondere auch, in welchem Umfang er vertragsärztlich tätig werden wolle. Wenn die BAG nur in geringem Umfang vertragsärztlich tätig geworden sei - aus welchen Gründen auch immer: sei es, dass ihnen der Patientenzulauf fehlte, dass ihnen die Zuweisungen von Patienten durch andere Ärzte fehlten, dass sie sich auf Privatpatienten konzentrierten, dass sie insgesamt nur geringfügig ärztlich tätig werden wollten oder dass sie ihren Schwerpunkt in Tätigkeiten für Krankenhäuser sahen -, so sei dies, so das BSG, ihrem Verantwortungsund Risikobereich zuzuordnen. Ihrem Risikobereich sei es auch zuzurechnen, wenn sich daraus aufgrund zwischenzeitlicher Neugestaltung der Honorierungssystematik honorarbegrenzende Auswirkungen für das Folgejahr ergäben. Worin allerdings ein Unterschied zu einem neuzugelassenen Arzt bestehen solle, stellte das BSG nicht fest. Denn auch dieser trifft mit dem Antrag auf Zulassung eine seiner Risikosphäre zuzurechnende Entscheidung.

Diese Sichtweise des BSG greift indes zu kurz. Zwar ist dem BSG darin zuzustim- 
men, dass die wirtschaftliche Ausrichtung einer radiologischen Gemeinschaftspraxis den Gesellschaftern zugerechnet werden kann. Die Änderung der Honorarsystematik liegt indes außerhalb des Verantwortungsbereiches der Radiologen. Ein Verschulden, welches eine Vorwerfbarkeit voraussetzt, kann nur vorliegen, wenn die Konsequenz, die das Verhalten nach sich ziehen würde, erkennbar gewesen war oder hätte erkannt werden müssen. Anhaltspunkte dafür finden sich keine, zumal sich in der Vergangenheit mehrfach zeigte, dass gesetzgeberische Änderungen des SGB V noch kurzfristig vor ihrer Verabschiedung durch den Gesetzgeber erfolgt sind.

Die Anerkennung eines Härtefalls setzt nach Ansicht des BSG neben der Existenzgefährdung der Praxis und einem bestehenden Sicherstellungsbedarf voraus, dass die maßgeblichen Umstände nicht von der Praxis zu vertreten sind. Das BSG verlangt daher einseitig die Solidarität der Praxis, wenn es auf den Sicherstellungsbedarf abstellt, verweigert der Praxis aber sogleich die Solidarität, wenn es zur Aufrechterhaltung der Sicherstellung auf diese Praxis nicht ankommt.

\section{Fazit}

$\nabla$

Die Entscheidung des BSG zeigt in einer bedauerlichen Offenheit, dass von dem niedergelassenen, vertragsärztlich tätigen Radiologen im Sinne seiner sozialen Verantwortung für das System der GKV verlangt werden kann, dass dieser Leistungen, die im Laufe eines Jahres erbracht werden, nicht vergütet erhält. Wenn der Vertragsarzt systemrelevant, also für die Sicherstellung von Bedeutung ist, kann ein Härtefall vorliegen und der Vertragsarzt erfährt seinerseits die Solidarität der GKV. Nun weigern sich die meisten Kassenärztlichen Vereinigungen vehement, die Bedeutung eines radiologischen Vertragsarztes für eine Region anzuerkennen. Kann dem Patienten doch zugemutet werden, dass er zu einem weiter entfernt ansässigen Radiologen anreist. Welche Folge dies z. B. auf die Wartezeiten der Patienten hat, wird in der aktuell aufgekommenen Überlegung, die Wartezeiten verbindlich und sanktionsbewährt zu regeln, nicht beachtet. An dieser Stelle befindet sich zugleich die unmittelbare Verbindung zwischen Honorierung und Sicherstellungsauftrag der Kassenärztlichen Vereinigungen. Diese Verbindung wird gelegentlich nicht von den Kassenärztlichen Vereinigungen gesehen und die Übermittlung statistischer Unterlagen zur Prüfung der Bedeutung einer Praxis für die Sicherstellung verweigert. Dabei zeigt die vorstehende Entscheidung des BSG, dass die vertragsärztliche Versorgung ein einheitliches System ist und die Honorarsystematik einerseits und die Sicherstellungssystematik nicht losgelöst voneinander zu betrachten sind. Die Kassenärztlichen Vereinigungen sind für beides verantwortlich und haben beide Aspekte zu berücksichtigen.

Nicht zu überzeugen vermag das BSG auch mit seiner Auffassung, dass es dem unternehmerischen Risiko des Vertragsarztes zuzurechnen sei, wie er seine Praxistätigkeit gestalte, insbesondere auch, in welchem Umfang er vertragsärztlich tätig werden wolle. Die Realität sieht anders aus, wie jeder niedergelassene, vertragsärztlich tätige Radiologe weiß. Die bestehende Honorarsystematik und die gelebte Praxis in den Kassenärztlichen Vereinigungen lassen ein Wachstum einer Praxis kaum zu und wer einmal ein Budget abgebaut hat, erlebt das Moratorium wieder und wieder. Die steten Änderungen der Honorarsystematik lassen daneben die Planungssicherheit für den vertragsärztlich tätigen Radiologen schwinden, obwohl er diese für die Investitionen in neue Großgeräte dringend benötigt. Dies alleine mit dem unternehmerischen Risiko des Radiologen zu begründen, ist nicht nur ein unnötiger Affront, es gefährdet die Sicherstellung der vertragsärztlichen Versorgung im Bereich der Radiologie. Eine Vielzahl von Aspekten wurde in der Entscheidung des BSG nicht erwähnt, so zum Beispiel worin die Begründung der Honorarzuwachsregelung aufgrund des Moratoriums bei einer überweisungsgebundenen medizinischen Fachdisziplin liegen soll. Den Radiologen auf fehlende Zuweisungen zu verweisen und ihm ein Verschulden für eine solche Situation anzulasten, müsste im Gegenzug heißen, dass das Einwirken auf mehr Zuweisungen durch andere Ärzte legitim sein müsste - was es aber regelmäßig nicht ist, zumindest wenn diese Einwirkung aus einem Entgelt besteht.

Das BSG wird sich auch zukünftig mit den Fragen der Honorierung auseinandersetzen müssen. Die vorliegende Begründung setzt sich nur mit einem kleinen, wenn auch relevanten Teil von Rechtsfragen auseinander. Der Gesetzgeber hat dane- ben die Regelungen zur Honorierung im SGB V verändert, sodass das BSG genau genommen über eine überholte Rechtslage urteilen musste. Zukünftige Entscheidungen können daher abweichen. In der Entscheidung des BSG werden die relevanten grundrechtlichen Regelungen des Gleichbehandlungsgebotes aus Art. 3 Abs. $1 \mathrm{GG}$ und der Berufsausübungsfreiheit aus Art. 12 Abs. 1 zwar erwähnt, jedoch hinsichtlich des Eingriffs und der zulässigen Schranken nur unzureichend gewürdigt. Über kurz oder lang, wird daher ein niedergelassener Radiologe den Gang zum Bundesverfassungsgericht nach Karlsruhe suchen und finden, um diese Fragen intensiv klären zu lassen.

\section{René T. Steinhäuser \\ Rechtsanwalt}

Rechtsanwälte Wigge

Neuer Wall 44

20354 Hamburg

Telefon: (040) 3398705-90

Telefax: (040) 3398705-99

Internet: www.ra-wigge.de

E-Mail: hamburg@ra-wigge.de 